ANA MARÍA ATTIÍAS

\title{
LA CAPACIDAD DE GESTIÓN DE LAS ÁREAS SOCIALES MUNICIPALES
}

La autora es arquitecta, (UNNE, Argentina), Auxiliar docente e investigadora (UNNE, Argentina) y Responsable de la Agencia CHACO de SIEMPRO -Sistema de Información y Monitoreo de Programas Sociales-

\begin{abstract}
RESUMEN. EI presente trabajo pretende arribar a una aproximación acerca de la capacidad de gestión de las áreas sociales de los municipios (ASM) de la Provincia del Chaco, tema que se transforma en relevante en el proceso creciente de descentralización de las políticas sociales, dejando en manos de los municipios la implementación y ejecución de las mismas, donde el modelo emergente tiende a privilegiar que las decisiones se tomen en el ámbito local. Para ello se analizaron ocho casos de estudio, conformados por municipios de primera, de segunda y de tercera categoría, donde se intentó medir la capacidad de gestión en función de las siguientes variables: el nivel de conformación de las unidades responsables del área social; la presencia de recursos humanos calificados; el grado de participación en la planificación, ejecución y evaluación de programas, proyectos y/o actividades; la disponibilidad del recurso financiero requerido; y el grado de articulación.
\end{abstract}

\section{ABSTRACT. COUNCILS' MANAGEMENT CAPABILITY OF SOCIAL DEPARTMENTS.}

This article is an exploratory approximation of the Chaco province' 'Municipal Social Areas Management Capabilities '(MSA). This theme grows in importance as the result of the decentralization process of social policies. The implementation of these policies has been transferred from national to municipal level. It attempts to empower local decision-making. This article took in consideration three municipal categories in order to measure their management capability, according to the following variables; 1-The formation of social units, 2- Skilled human resources, 3- The involvement in the development of programmed-projects or related activities, considering, planning, implementation, evaluation, 3-Financial support required, 4- Articulation level.

\section{LAS ÁREAS SOCIALES MUNICIPALES Y EL PROCESO DE DESCENTRALIZACIÓN DE LA POLÍTICA SOCIAL}

En América Latina se han iniciado procesos de cambio que propugnan transferencia de competencias hacia los municipios, las cuales hacen que su capacidad de gestión se vea alterada. Esta transferencia de competencias se enmarca en una política de descentralización iniciada en Argentina en la década de los noventa, donde las acciones en esta dirección tienen un ritmo progresivo.

No hay duda que las transferencias de funciones desde los niveles centrales de gobierno, de la nación, y de la provincia a los municipios, es sin duda el paradigma de la descentralización.

Desde el nivel local esta perspectiva implica no solamente un nuevo espacio de responsabilidades, sino también una oportunidad de decisión sobre los modos de ejercicio de tales responsabilidades. La modalidad de implementación se encuentra en el margen de los grados de libertad del gobierno local para la asunción de las nuevas responsabilidades, necesariamente determinadas desde la política central de descentralización.

En el presente artículo la descentralización tiene que ver con la transferencia de competencias y recursos de un nivel central de gobierno, tanto nacional como provincial, hacia otro de distinto origen y que guarda autonomía, los municipios. La ampliación de responsabilidades de los gobiernos locales que resulta del ejercicio de los nuevos roles y funciones que progresivamente van asumiendo.

El análisis de las capacidades del municipio para responder a la misma es el principal problema al considerar la viabilidad y sustentabilidad de la descentralización. Paralelamente a este proceso de descentralización en la ejecución de las políticas sociales hacia los gobiernos locales, la capacidad de gestión de las áreas sociales municipales históricamente ha estado limitada, llegándose a situaciones 
críticas en las que muchos de los Municipios ni siquiera cuentan con la existencia de estas áreas dentro de la estructura municipal.

Estas tienen muchas restricciones respecto de las acciones más estructurales, razón por lo cual es aún mayor su dificultad cuando se pretende que inicien procesos tales como la participación ciudadana en las formas de gestión o la incorporación de modalidades asociativas para promover la gobernabilidad local, impuestas de manera vertical como condición para garantizar la continuidad de algunos programas sociales desde niveles centrales del Estado.

El inicio de la nueva gestión del gobierno nacional, incorporó a la política social nuevas modalidades en la implementación de los programas sociales que recientemente iniciaron su ejecución en nuestra provincia, nos referimos a programas nacionales de financiamiento internacional. La innovación consiste en que estos "programas", manejan los conceptos de integralidad de prestaciones, articulación de actores, ejecución descentralizada, desarrollo local, gestión asociada y participación ciudadana, entre otros. Este modelo de gestión es también replicado por los propios programas provinciales, que propugnan la instalación de este modelo de gestión social.

Este trabajo intenta hacer un aporte en el impacto que causa la descentralización en los municipios, más precisamente en sus áreas sociales, tratando de analizar acerca de cómo se encuentran éstas preparadas para enfrentar el nuevo cuadro de situación, las que deben hacerse cargo de la ejecución de las acciones descentralizadas desde el gobierno nacional y provincial.

La hipótesis que guía el presente trabajo sostiene que en el complejo marco de las competencias y funciones de los gobiernos locales, efecto de un alto grado de descentralización, así como las crecientes presiones y exigencias de la propia comunidad, han ensanchado la brecha entre la gestión y la demanda, lo que ubica a las áreas sociales municipales en la necesidad de mejorar su capacidad de gestión de manera inmediata.

Que las áreas sociales municipales de la Provincia del Chaco, no se encuentran lo suficientemente consolidadas como para seguir recibiendo programas sociales, si antes no se hace un esfuerzo por su afianzamiento; y que de no realizarse un esfuerzo para su fortalecimiento, ante la progresiva descentralización de funciones, tanto de la nación como de la provincia, las mismas no podrán en un futuro mediato implementar las acciones de manera eficiente y eficaz.

Las demandas de la población son diversas, y las respuestas para intentar aliviarlas también son de diferentes tipos, la existencia de una multiplicidad de programas sociales focalizados hacia el sector de extrema pobreza, que van desde las ayudas directas a la población de menos recursos a la implementación de programas que persiguen distintos fines, involucran a las áreas sociales municipales en el manejo de temas variados, tales como el alimentario, la tramitación de pensiones hasta el saneamiento urbano, entre otros.

Es así como las áreas sociales municipales, sus intendentes o presidentes de los Consejos Municipales y equipos de gestión, requieren desarrollar cierta solvencia en el tratamiento e implementación de esas respuestas.

Por lo que, es necesario revisar la situación de las áreas sociales municipales en relación con sus capacidades de gestión y los escenarios en materia de descentralización. Procesos que vinculados con la característica de la población, ayuden a vislumbrar necesidades en cuanto a la generación y manejo de información, capacitación y fortalecimiento, con lo cual puedan mejorarse las capacidades decisionales para actuar sobre cada territorio.

Los Municipios se enfrentan diariamente a una dinámica compleja y cambiante en su entorno sociopolítico y económico. Dentro de este complejo cuadro de situación, este trabajo propuesto se centrará en la realidad actual de las áreas sociales municipales en la Provincia del Chaco, tema que se transforma en relevante en el proceso creciente de descentralización de las políticas sociales, dejando tanto la provincia como la nación, en manos de los Municipios su implementación y ejecución, donde el modelo emergente tiende a privilegiar que las decisiones se tomen en el ámbito local.

El presente trabajo tiene como objetivo central analizar la capacidad de gestión de las áreas sociales de determinados Municipios de la Provincia del Chaco, poniendo el énfasis en su conformación y 
grado de consolidación, de modo de detectar sus problemas, demandas, limitaciones y potencialidades en relación con el reciente proceso de descentralización de la política social hacia los gobiernos locales.

Respecto de las áreas sociales municipales en nuestra Provincia, mas precisamente en relación con las necesidades de gestión y al desempeño de los gobiernos locales, existe un gran vacío de información. La única forma de poder subsanar esta situación, es a través del relevamiento de experiencias específicas, para que podamos contar con la información necesaria para elaborar recomendaciones acerca del mejoramiento del gerenciamiento de estas áreas, el diseño de estructuras organizativas apropiadas y la capacitación de los equipos técnicos municipales.

Los principales vacíos del problema residen en que si bien abundan los estudios y publicaciones respecto de los efectos de la política de globalización y descentralización, y acerca de su impacto sobre los gobiernos locales, es importante sobre todo en esta área del saber la profundización en estudios particulares y específicos de los gobiernos municipales.

La profundidad y fortaleza del presente trabajo reside que en él se estudian casos específicos de la capacidad de gestión de las áreas sociales de gobiernos locales, abordándose elementos propios de la gestión de éstas áreas en el marco de las experiencias y procesos de cambios que se han venido produciendo dentro de los Municipios de la Provincia del Chaco.

\section{DETERMINACIÓN DE VARIABLES}

La capacidad de gestión está conformada tanto por variables vinculadas a sus características particulares, las estructuras, los procesos y los recursos disponibles, como también por otras tales como factores externos, el entorno económico, político y social en el cual cada gobierno desarrolla sus actividades. Debe considerarse el contexto institucional del sector público, los recursos y las estructuras formales e informales de influencia, y es necesario tener en cuenta la red de organizaciones y actores vinculados a la implementación de las políticas y sus modos de articulación.

Esta propuesta metodológica intenta incluir el análisis del entorno institucional externo, el de las relaciones interinstitucionales y el análisis del entorno micro, aspectos relacionados a la organización interna, a la asignación de funciones y de recursos, el personal y las capacidades individuales.

Entendiendo por capacidad a la habilidad de desarrollar tareas de manera eficiente y eficaz, por lo que la capacidad de gestión puede ser analizada a través del estudio de variables asociadas a su desempeño. Lo que implica tomar aspectos ligados a los procesos internos, tales como estructura, recursos humanos, financieros y políticos y prácticas internas; sino también a aquellos vinculados con los modos en que la institución se vincula con otros actores gubernamentales y no gubernamentales.

Algunas de las variables que contribuyen a arribar a una aproximación acerca de la capacidad de gestión de las áreas sociales municipales, son las siguientes:

1. El nivel de conformación de las unidades responsables del área social.

2. La presencia de recursos humanos calificados.

3. El grado de participación en la planificación, ejecución y evaluación de programas, proyectos y/o actividades.

4. La disponibilidad del recurso financiero requerido.

5. El grado de articulación.

\section{LA DEFINICIÓN DEL UNIVERSO DE OBSERVACIÓN}

Si bien la totalidad de Municipios de la Provincia del Chaco asciende a 68, el número de Municipios que representa el Universo para el presente trabajo estuvo acotado respecto de su número, pero no en la profundidad de su estudio. La determinación del Universo de los Municipios que se conformarían como casos de estudio, se realizó en función de los siguientes criterios: 
n Que los Municipios pertenezcan a las tres categorías establecidas por el régimen municipal de la Provincia del Chaco, de primera, de segunda y de tercera.

n Que en los Municipios se hubiere implementado el Relevamiento SISFAM (Registro Único de Familias Beneficiarias y Potenciales de Programas Sociales), de modo de que tengan una relación previamente establecida con el Programa SIEMPRO, lo que garantizara un más rápido y eficiente intercambio de información.

n Que los Municipios hubieran manifestado interés, en que el SIEMPRO, realice el estudio de la capacidad de gestión de las áreas sociales respectivas.

Por que el Universo del trabajo propuesto se conformó por ocho Municipios, considerados como casos de estudio.

Cuadro 1: Casos de estudio

\begin{tabular}{lll}
\hline Categoría & Municipio & Departamento \\
\hline Primera & Barranqueras & San Fernando \\
\hline Segunda & Fontana & San Fernando \\
& Puerto Vilelas & San Fernando \\
& La Leonesa & Bermejo \\
& Pampa del Indio & Libertador General San Martín \\
\hline Tercera & Basail & San Fernando \\
& Colonia Benítez & 1 de Mayo \\
& Margarita Belén & 1 de Mayo \\
& \\
\end{tabular}

Fuente: Secretaría de Asuntos Municipales, Marzo 2001, Elaboración propia.

\section{MODALIDADES OPERATIVAS}

En un Primer nivel de investigación se realizaron entrevistas a los responsables e integrantes de los equipos técnicos de las áreas sociales de cada uno de los Municipios objeto de estudio; y se analizó el comportamiento de cada una de las variables de la capacidad de gestión de las áreas sociales municipales en los casos de estudio.

En un Segundo Nivel se intentó medir en cada uno de los casos de estudio el comportamiento de cada variable en sus diferentes aspectos analizados, a través de su ponderación con una escala de valores de cero (0) a cinco (5), donde el cero corresponde al valor mínimo que adquiere cada variable o sea nula capacidad de gestión y cinco, el valor máximo u óptimo de la misma, es decir, alta capacidad de gestión. Se obtuvo un valor promedio de cada una de las variables para cada Municipio caso de estudio y se realizó un cruce de los valores síntesis de cada una de las variables, es decir un valor promedio de las cinco variables consideradas relevantes en cada Municipio, para así determinar finalmente la capacidad de gestión de las áreas sociales municipales.

\section{FUENTES DE INFORMACIÓN UTILIZADAS}

Una de las mayores limitaciones para implementar el trabajo estuvo dado por la inexistencia en ningún área u organismo provincial de la información necesaria acerca de estudios específicos a nivel municipal. No se pudieron consultar fuentes secundarias de información, ya que el tipo de información de que se requería no estaba registrada. La fuente de información más importante fueron las entrevistas efectuadas a los responsables de las áreas sociales municipales, a los intendentes y/o presidentes de concejos municipales, según una guía diseñada para este fin, las que fueron realizadas durante el período comprendido entre los meses de Enero a Marzo del 2001. 


\section{LA CAPACIDAD DE GESTIÓN DE LAS ÁREAS SOCIALES MUNICIPALES. ANÁLISIS DEL COMPORTAMIENTO DE CADA UNA DE LAS VARIABLES}

\section{El nivel de conformación de las unidades responsables del área social:}

La conformación de las áreas sociales son las respuestas que los Municipios emiten ante demandas específicas que le competen. La lógica del desempeño institucional establece que, los gobiernos locales deben instaurar instancias que absorban y ejecuten las demandas específicas en el ámbito social, generadas a partir de una política de descentralización desde el Estado Nacional y/o Provincial. Su creación debe estar acompañada de la dotación de los recursos apropiados que garantice su funcionamiento.

La totalidad de los casos de estudio tienen dentro de la estructura municipal una unidad responsable del área social. La creación de estas unidades surge a modo de respuesta de parte de los Municipios, ante la creciente descentralización de acciones y de recursos del estado nacional como provincial. En su totalidad son de reciente creación, las que tienen dentro de la estructura municipal diferentes rangos desde Secretarías hasta Direcciones, variando las tipologías de inserción de las áreas sociales en el organigrama municipal, de acuerdo al rango éstas adquieren.

Cuadro 2: ASM en los casos de estudio

\begin{tabular}{llllll}
\hline Municipio & $\begin{array}{l}\text { Área } \\
\text { Social }\end{array}$ & Rango & Denominación & $\begin{array}{l}\text { Dependencia } \\
\text { administrativa }\end{array}$ & $\begin{array}{l}\text { Fecha } \\
\text { creación }\end{array}$ \\
\hline Barranqueras & Sí & Dirección & Dirección de Acción Social & Secretaría de Gobierno & 1999 \\
\hline Fontana & Sí & Secretaría & Secretaría de Acción Social & Intendente & 1999 \\
\hline Puerto Vilelas & Sí & Subsecretaría & Subsecretaría de Desarrollo Social & Intendencia & 1995 \\
\hline La Leonesa & Sí & Departamento & Departamento de Acción Social & Secretaría de Gobierno & 2000 \\
\hline Pampa del Indio & Sí & Secretaría & Secretaría de Acción Social & Intendencia & 1999 \\
\hline Basail & Sí & Secretaría & Secretaría de Trabajo, & Intendencia & 1999 \\
& & & Producción y Desarrollo Social & & \\
\hline Colonia Benítez & Sí & Secretaría & Secretaría de Acción Social & Intendencia & 1999 \\
Margarita Belén & Sí & Secretaría & Secretaría de Acción Social & Concejo Municipal & 1994 \\
\hline
\end{tabular}

Fuente: Entrevista realizada a responsables municipales, Marzo 2001. Elab. propia.

En los casos analizados, las áreas sociales tienen el rango de secretaría en los Municipios de tercera categoría, esta situación disminuye en los de segunda y en el municipio de primera tiene un rango de menor jerarquía. Esto quizás evidencia el alto impacto que tiene sobre las estructuras organizativas de los municipios más pequeños la descentralización de la política social a escala local. La jerarquización de las áreas sociales en estos municipios representa un reconocimiento a la importancia que los programas tienen en relación con la satisfacción de las necesidades básicas de los sectores más postergados de sus comunidades.

La estructura organizativa funcional responde al siguiente esquemas de áreas: atención de la demanda directa de la población, tramitación de pensiones y jubilaciones, programas alimentarios, y un área donde se realizan las tareas administrativas relativas a las erogaciones realizadas.

Si en el Municipio se implementan programas sociales de gran envergadura, tanto por la complejidad de las acciones que demanda como de la importancia de los recursos incorporados al municipio se crea un área al efecto.

Los Municipios de tercera categoría no poseen áreas para su funcionamiento interno, al estar éstas conformadas por solamente dos personas, el esquema de organización varía sustancialmente, constituyendo instituciones en que el personal ejerce roles múltiples, manteniéndose el tipo de acciones que desarrollan. 
Otro aspecto a considerar está dado no sólo por la creación de un área responsable en lo social, sino paralelamente la dotación de recursos materiales necesarios para su funcionamiento, tales como equipamiento, movilidad y disponibilidad de medios de comunicación, como así también de un espacio físico adecuado para el desarrollo de las actividades inherentes al área (atención de personas, archivo de material, reuniones y trabajo en equipo, etc). La dimensión de estos recursos nos otorga la posibilidad de constatar la realidad de estas áreas, y poder tener una visión clara de las limitaciones que tienen.

Respecto de la disponibilidad del equipamiento informático, se pudo verificar que la mayoría de los casos disponen de una computadora de uso exclusivo del área, ya que en los Municipios considerados como casos de estudio se implementó un programa nacional que les aportaba una computadora para el área social.

En relación con la disponibilidad de movilidad, la situación se repite en casi la totalidad de los casos, los Municipios disponen de un solo vehículo para todas las áreas del gobierno municipal. Este dato no debe ser considerado menor, ya que varios de los programas sociales en ejecución se ejecutan en diferentes barrios y colonias distantes.

Respecto de la disponibilidad de medios de comunicación, se pudo observar que en este aspecto la situación de la totalidad de las áreas sociales, es dramática. La descentralización de programas sociales de gran envergadura puede convertirse en una mera ficción si el Municipio no tiene ningún medio de comunicación con el exterior (teléfono, fax o Internet). En la totalidad de los casos los municipios disponen de un único teléfono, ninguno de los casos cuenta con fax; y un solo municipio utiliza Internet.

La disponibilidad de un espacio físico reducido e insuficiente para el desarrollo del área social tiene también su influencia sobre su capacidad de desempeño. La situación de la mayoría de los casos en este aspecto es igualmente crítica, los espacios son reducidos e inadecuados, lo que sumado a una imposibilidad de poder contar con medios de comunicación, ni movilidad, tiene una influencia decisiva en su funcionamiento.

\section{La presencia de recursos humanos calificados:}

La calificación del personal que integran los equipos técnicos locales constituye un claro indicador de la capacidad de gestión de las áreas sociales. Esta variable representa un factor clave en el desempeño organizacional de éstas áreas. Por lo que es necesario que se tenga en cuenta, ante la creciente descentralización de programas sociales hacia los municipios, la constitución y grado de solidez que tienen los equipos técnicos municipales.

En general, las áreas sociales cuentan con equipos técnicos reducidos, en relación con la multiplicidad de tareas que desempeñan y en comparación con la totalidad de empleados municipales. La cantidad de personal que integran las áreas sociales no responde a que a mayor cantidad de población a atender mayor cantidad de personal, ya que el municipio de primera categoría, tiene menor cantidad de personal que los de segunda.

Cuadro 3: Cantidad de personas que integran los equipos técnicos de las ASM y relación con la totalidad de personal municipal

\begin{tabular}{llll}
\hline Municipio & $\begin{array}{l}\text { Cantidad de personas } \\
\text { del área social }\end{array}$ & $\begin{array}{l}\text { Total de personal } \\
\text { municipal, de planta } \\
\text { y contratados }\end{array}$ & $\begin{array}{l}\% \text { personal del área social } \\
\text { sobre total del personal } \\
\text { total del pers. del municipio }\end{array}$ \\
\hline Barranqueras & 6 & 522 & 1.14 \\
\hline Fontana & 7 & $\begin{array}{l}146 \quad \text { (pers. } \\
\text { planta) }\end{array}$ & de \\
\hline Puerto Vilelas & 11 & 206 & 5.79 \\
\hline La Leonesa & 4 & 90 & 4.44 \\
\hline Pampa del Indio & 5 & 70 & 7.14 \\
\hline
\end{tabular}




\begin{tabular}{llll} 
Basail & 2 & 30 & 6.66 \\
\hline Colonia Benítez & 2 & 35 & 5.71 \\
\hline Margarita Belén & 2 & 43 & 4.65 \\
\hline
\end{tabular}

Fuente: Entrevista realizada a responsables municipales, Marzo 2001. Elab. Propia.

En la mayoría de los equipos técnicos, al personal que es de la planta de empleados municipales, se le asignan roles con casi nula capacidad de decisión, tareas administrativas menores y rutinarias. Una proporción mínima de ellos cumple roles técnicos de mayor envergadura y poder de decisión, tales como jefe de departamento y referentes de programas nacionales. Paralelamente podemos señalar que el porcentaje de empleados de planta tiene un bajo a muy bajo nivel educativo, en su mayoría el nivel alcanzado es primaria completa y secundario incompleto. El mayor poder de decisión lo tiene el personal contratado que ingresa con la nueva gestión, evidenciándose en algunos casos ciertas resistencias y desconfianza entre ellos.

Cuadro 4: Perfil de los integrantes de los equipos técnicos de las ASM (Máximo nivel educativo alcanzado).

\begin{tabular}{llllll}
\hline Municipio & \multicolumn{5}{c}{ Nivel de Formación } \\
\cline { 2 - 6 } & Universitario & Terciario & $\begin{array}{l}\text { Secundario } \\
\text { completo }\end{array}$ & $\begin{array}{l}\text { Secundario } \\
\text { incompleto }\end{array}$ & Primario completo \\
\hline Barranqueras & 0 & 0 & 2 & 4 & 0 \\
\hline Fontana & 0 & 1 & 1 & 0 & 5 \\
\hline Puerto Vilelas & 1 & 3 & 3 & 0 & 4 \\
\hline La Leonesa & 0 & 0 & 2 & 2 & 0 \\
\hline Pampa del Indio & 0 & 2 & 0 & 3 & 0 \\
\hline Basail & 0 & 0 & 2 & 0 & 0 \\
\hline Colonia Benítez & 0 & 0 & 1 & 1 & 0 \\
\hline Margarita Belén & 0 & 0 & 1 & 1 & 9 \\
Totales & 1 & 6 & 12 & 11 & \\
\hline
\end{tabular}

Fuente: Entrevista realizada a responsables municipales, Marzo 2001. Elab. propia.

Los programas nacionales en su estrategia de descentralización refuerzan los equipos locales, contratando personal con cierta calificación (nivel universitario y/o terciario, personal con conocimiento en informática u otras especializaciones según las prestaciones que realizan) como sus referentes en el ámbito local.

La situación de prácticamente la mitad de los integrantes de los equipos técnicos de las áreas sociales, responde a contrato, bajo diversas modalidades, contratos de servicio y/o de obra, subsidios al empleo; y por personal afectado en forma temporaria de otras áreas del mismo municipio y en casos extremos, algunos integrantes tienen una dedicación sin ningún tipo de remuneración (ad-honorem). El personal de planta sólo alcanza un 41\%. Esto crea una gran inestabilidad en los equipos técnicos, que sumado a bajas remuneraciones, genera un cuadro de incertidumbre en el ámbito municipal acerca de la posibilidad de cumplimiento de los compromisos contraídos, convenios y acuerdos firmados con la Nación como con la Provincia para la ejecución de los programas sociales en el ámbito local.

De esta modalidad de funcionar con equipos, casi en su totalidad bajo alguna forma de contrato, los integrantes en su mayoría tienen como máximo dos años de antigüedad. Esta situación contractual permite que cada cuatro años, los integrantes de los equipos sean removidos por las nuevas autoridades, impidiendo de esta manera la continuidad del personal en el área, perdiendo el municipio toda su experiencia y formación, para que en cada caso se vuelva a empezar prácticamente de nuevo en la capacitación del personal. 
El perfil de los responsables de las áreas sociales municipales en los casos de estudio, en relación con su formación y estudios cursados es la siguiente: ningún responsable es profesional universitario; tres tienen estudios de nivel terciario, una asistente social y dos docentes; tres tienen secundario completo y dos secundario incompleto, donde la mayoría de los responsables provienen de la militancia política en el ámbito local en distintos partidos, el cargo no responde a la existencia o vigencia de una carrera pública, sino que se considera un cargo de naturaleza política.

\section{El grado de participación en la planificación, ejecución y evaluación de programas, proyectos y/o actividades:}

El desarrollo de las capacidades apropiadas para la planificación, ejecución y evaluación de planes, programas y/o proyectos destinados al sector de extrema pobreza representa para los Municipios en general, y para las áreas sociales en particular un alto valor institucional. La posibilidad de poder incorporar al Municipio la implementación de programas y/o proyectos sociales se convierte, en un medio para innovar y captar nuevos recursos, ya sean provenientes del estado nacional como provincial, lo que adquiere una dimensión significativa de ingreso de recursos de origen externo en la canalización de la demanda local.

La detección de cadenas de responsabilidades en la ejecución de los planes, programas y proyectos sociales; las acciones y tareas a cargo de los Municipios; los deberes, compromisos y obligaciones en el cumplimiento del rol que le confiere el nuevo modelo de gestión de programas sociales, se convierten en un elemento clave para intentar medir su capacidad.

Con relación a la existencia de capacidad técnica para planificar en las áreas sociales municipales, más precisamente para la formulación de programas y/o proyectos es prácticamente nula, sólo un municipio tiene formulado un programa municipal, lo que habitualmente formulan los equipos técnicos municipales son los proyectos de los programas de empleo.

En los casos de estudio no existen documentos escritos acerca de la planificación de las actividades de las áreas sociales. Solo cuentan con resoluciones municipales, donde se definen misiones y funciones. Las áreas sociales tienen una organización interna que se establece en función de la demanda, tanto de la propia población como de los organismos provinciales y nacionales con quienes se articulan en la implementación de programas.

Actualmente éstas perciben la necesidad de contar con bases propias de información social que registren las demandas, las prestaciones ejecutadas con la identificación de los beneficiarios, de modo de poder disponer de información para la toma de decisiones. Las áreas sociales intentan implementar alguna forma de registro de sus beneficiarios y de las prestaciones realizadas a través de la asistencia a la demanda espontánea (bolsas de mercaderías, subsidios económicos, medicamentos, etc) de manera de manera de poder planificar el uso de sus escasos recursos. Los registros al momento de este estudio son imperfectos, poco sistemáticos y no informatizados.

Respecto del modelo de gestión de las áreas sociales municipales, se pudo verificar que basan su actividad en la realización de funciones administrativas y de gestión. En algunos la gestión consiste en atender a la demanda espontánea de los pobladores más carenciados, según la disponibilidad de sus propios recursos. La asistencia directa canalizada con este tipo de recursos, es de exclusiva responsabilidad de los municipios, donde se decide quiénes son los beneficiarios, la estipulación de la asignación, etc.

Básicamente las actividades realizadas por las áreas sociales municipales consisten en:

n Entrega de bienes y/o servicios a población pobre: bolsas de mercaderías, indumentaria o ropa de abrigo, elementos para la construcción de ranchos (postes, chapas, clavos), provisión de ataúdes y material para la construcción de bóvedas, pasajes para traslados, medicamentos y fotografías para la tramitación de DNI, entre otros. 
n Entrega de subsidios económicos: subsidios económicos a personas físicas con un alto grado de necesidad y a instituciones locales.

n Asistencia a población de escasos recursos ante situaciones de emergencia, tales como asistencia a inundados.

La recepción de la demanda espontánea, la canalización y la tramitación de los bienes y/o servicios ante organismos provinciales, son las acciones centrales desplegadas desde las áreas sociales de jurisdicción municipal. Es el Intendente quien es la figura política, que negocia y gestiona hacia fuera, y en su capacidad reside que el municipio sea focalizado para la implementación de programas sociales.

Cuadro 5: Número de programas sociales nacionales y provinciales en que las ASM de los casos de estudio participan en su ejecución.

\begin{tabular}{|c|c|c|c|c|}
\hline Municipio & $\begin{array}{l}\text { Número total de } \\
\text { programas que se } \\
\text { ejecutan en el } \\
\text { Municipio }\end{array}$ & $\begin{array}{l}\text { Número de programas } \\
\text { nacionales que se } \\
\text { involucran a las áreas } \\
\text { sociales municipales }\end{array}$ & $\begin{array}{l}\text { Número de programas } \\
\text { provinciales que } \\
\text { involucran a las áreas } \\
\text { sociales municipales }\end{array}$ & $\begin{array}{l}\text { \% de programas externos } \\
\text { con participación en la } \\
\text { ejecución de las áreas } \\
\text { sociales municipales }\end{array}$ \\
\hline Barranqueras & 20 & 8 & 5 & $65 \%$ \\
\hline Fontana & 24 & 9 & 5 & $58 \%$ \\
\hline Puerto Vilelas & 16 & 6 & 4 & $63 \%$ \\
\hline La Leonesa & 16 & 5 & 3 & $50 \%$ \\
\hline Pampa del Indio & 13 & 3 & 3 & $46 \%$ \\
\hline Basail & 14 & 7 & 3 & $71 \%$ \\
\hline Colonia Benítez & 12 & 6 & 3 & $75 \%$ \\
\hline Margarita Belén & 14 & 5 & 4 & $64 \%$ \\
\hline
\end{tabular}

Fuente: Entrevista realizada a responsables municipales, Marzo 2001. Elab. propia

De la totalidad de programas y/o proyectos sociales nacionales y provinciales que se implementan en los casos de estudio, según el número de programas como así también de la cantidad de recursos que aportan, de la cantidad de prestaciones ejecutadas y del número de beneficiarios atendidos, podemos confeccionar el siguiente orden de importancia, a saber: programas alimentarios; programas de integración y desarrollo comunitario; programas de vivienda e infraestructura social; programas de empleo y desarrollo productivo; programas de subsidio; y en último lugar, programas de capacitación y fortalecimiento.

La totalidad de los programas de origen externo a los municipios, descentralizan exclusivamente la ejecución de las acciones necesarias para su implementación. El grado en que los diferentes programas involucran a las áreas sociales municipales varía, ya que algunos solo descentralizan algunas acciones puntuales y acotadas, mientras que otros, los que responden al nuevo modelo de gestión de la política social, aspiran a un mayor grado de descentralización de la ejecución de las acciones necesarias para su implementación.

Ninguno de los programas descentralizan ninguna actividad ni tarea relacionada con la planificación de los mismos, ni tampoco delegan ninguna actividad relacionada con su evaluación. Si bien algunos tienden a descentralizar en las áreas sociales municipales algunas tareas relacionadas con la supervisión de las prestaciones, actividad relacionada con el contralor de su implementación, actividad meramente instrumental, que con la instalación de una práctica de seguimiento y evaluación que permitan retroalimentar la acción. No existe práctica en el seguimiento y evaluación de las acciones implementadas por las áreas sociales municipales.

\section{La disponibilidad del rec urso financiero requerido:}


La capacidad de las áreas sociales de disponer del recurso financiero necesario para su funcionamiento y para el desarrollo de programas, proyectos y/o actividades previstas en el tiempo y forma requerido, ante el dramatismo de la situación social, que en la mayoría de los casos requiere una urgente resolución, es un indicador tan obvio como el de recursos humanos calificados. Este factor para los gobiernos municipales, es decisivo respecto de su capacidad de gestión para responder a las demandas locales.

Para comprender mejor el contexto de las áreas sociales municipales, es necesario hacer referencia a la dramática situación financiera por la que están atravesando los municipios. Podemos afirmar que la totalidad de los municipios se encuentran en un cuadro de fuerte desfinanciamiento, agravado en algunos casos por mantener una relación conflictiva con el personal municipal, al que adeudan salarios atrasados. Algunos se encuentran en una instancia de reordenamiento administrativo y financiero, con una marcada dependencia de los aportes especiales o extraordinarios del gobierno provincial.

Los municipios objeto de estudio tienen un alto déficit, algunos de ellos cuentan con una deuda histórica que están pagando y que les consume casi la totalidad de los recursos propios, agravado en algunos casos por la disminución en los ingresos debido al cierre de industrias que constituían la fuente de su recaudación.

La alta dependencia de los recursos extra-municipales, es decir de los ingresos provenientes de programas provinciales y nacionales le otorga como consecuencia a las áreas sociales, una extrema debilidad ante eventuales situaciones de crisis temporaria u otras situaciones externas. Esto acentúa el riesgo ante posibles situaciones de incumplimiento, ya que grandes sectores carenciados de la población son asistidos por programas, por ejemplo los programas alimentarios cuyo origen de financiamiento es nacional y/o provincial.

En muchos casos el recurso financiero necesario para el funcionamiento de las actividades previstas y los compromisos contraídos por las áreas sociales, no lo reciben ni en el tiempo ni en la forma prevista. Es moneda corriente que los programas tanto nacionales como provinciales no envíen a los Municipios las entregas de las prestaciones comprometidas (bolsas y cajas de mercaderías, asignaciones previstas, pago de becas, etc) en el tiempo estipulado (según plazos de entrega, mensual, bimestral, etc) ni en la forma prevista (cantidad y tipo de mercaderías, montos acordados, etc), lo cual condiciona la operatividad del municipio haciéndolo altamente dependiente de los ingresos de otras jurisdicciones de financiamiento.

Cuadro 6: Programas sociales según el origen de su financiamiento, que involucran en su ejecución a los municipios casos de estudio

\begin{tabular}{ll}
\hline Programas Nacionales & Programas Provinciales \\
\hline UNIDOS & PRONU-CHACO \\
\hline PENSIONES NO CONTRIBUTIVAS & AIPO - PROGRAMA ALIMENTARIO \\
\hline SIEMPRO - SISFAM & AIPO - SIEMBRA VIDA \\
\hline PROGRAMA DE EMERGENCIA LABORAL & AIPO - MI TECHO \\
\hline TRABAJAR III & PROGRAMA EMERGENCIA \\
\hline JEFAS DE HOGAR & PLAN HACER \\
\hline SOLIDARIDAD & PENSIONES GRACIABLES \\
\hline FOPAR & PLAN DE ACCION COMUNITARIA \\
\hline PROG. DE PROTECCIÓN CONTRA LAS INUNDACIONES & \\
\hline MEJORAMIENTO DE BARRIOS & \\
\hline FONAVI & \\
\hline
\end{tabular}

Fuente: Entrevista realizada a responsables municipales, Marzo 2001. Elab. Propia. 
En consecuencia, esta situación de fragilidad estructural desde el punto de vista del financiamiento de las políticas sociales municipales, tiene una gran incidencia sobre la capacidad de gestión de las áreas sociales.

Por otro lado, igualmente negativa para el funcionamiento de las áreas sociales es la baja participación de los ingresos de la propia jurisdicción en la implementación de las políticas sociales municipales. Los municipios no cuentan con los fondos mínimos necesarios para paliar la situación de pobreza que está viviendo un alto porcentaje de la población. Un común denominador de las áreas sociales es que los recursos de que disponen son insuficientes.

De los ocho casos de estudio, las áreas sociales que tienen presupuesto propio, aunque limitados y escasos, son solamente dos municipios cuyo origen es de recurso genuino de los municipios. Son tres municipios cuyas áreas sociales que no tienen recurso propio, pero si disponen de un monto mensual fijo o variable entregado por el área de Tesorería, cuyo origen también proviene del recurso propio.

Las restantes áreas sociales no disponen en el presupuesto municipal ningún tipo de fondo para las erogaciones que les demanden éstas áreas, las que se desempeñan exclusivamente con fondos de origen externo. Los mecanismos de obtención de los fondos necesarios para su funcionamiento, es la canalización de la totalidad de las demandas recibidas ante la Secretaría de Desarrollo Social de la provincia.

La cantidad de recurso destinados a las áreas sociales, ya sea proveniente de recurso disponible por el área o asignado mensualmente por la tesorería del municipio, varía entre 1.000 y 4.000 pesos aproximadamente.

Respecto del destino de los recursos, en la totalidad de los casos de estudio se ha podido verificar que son para dar respuesta a la asistencia directa a la población carenciada de la comunidad, en prestaciones similares (ropa, medicamentos, alimentos, traslados, materiales para la construcción de ranchos, etc).

Esta crítica situación financiera tiene varias consecuencias, las áreas sociales se ven forzadas a canalizar la demanda espontánea proveniente de la asistencia directa de personas, hacia distintos programas u áreas del gobierno provincial para poder satisfacerla; al no disponer el área social municipal de fondos propios no puede planificar ningún tipo de acción; parte del equipo técnico debe abocarse a la gestión y resolución de la situación problemática que dio origen a la demanda, convirtiéndose en meros gestores de la política social.

En relación con que el Municipio tenga o esté en vías de tener algún programa municipal, los recursos con los que cuentan para su implementación no son recursos propios, sino de origen externo, sólo un pequeño porcentaje corresponde a recursos locales. Considerándolo desde este punto de vista la formulación de programas municipales tiene como objetivo la posibilidad de captar mayor ingreso de recursos de "afuera" bajo esta modalidad o por esta vía, lo que aumenta aun más su dependencia económica amparado bajo una falsa visión de desarrollo local.

\section{El grado de articulación}

La existencia de instancias de articulación formal de las áreas sociales municipales con organismos del estado provincial y nacional; con organismos no gubernamentales internacionales, nacionales, provinciales y/o locales; con organizaciones de la sociedad civil y su pertenencia a redes sociales, es una variable relevante para medir su capacidad de gestión.

El nuevo modelo de gestión de programas sociales, tanto nacionales como provinciales, tiene el acento en la articulación a escala local de los actores relevantes para la ejecución, la fiscalización y el control social. Las áreas sociales municipales según este nuevo modelo, deben articularse y de hecho lo hacen con los diferentes actores sociales locales para la implementación de los diferentes programas; articulaciones que varían de acuerdo a las prestaciones brindadas por los mismos, tales como, las diferentes iglesias, las organizaciones vecinales o comunitarias locales, los centros de jubilados, los efectores de salud, educación, y la policía, entre otros. 
Cuadro 7: Articulación formal de las ASM en los casos de estudio con organismos gubernamentales provinciales y nacionales, y organizaciones locales.

\begin{tabular}{|c|c|c|}
\hline Organismos gubernamentales nacionales & $\begin{array}{l}\text { Organismos gubernamentales } \\
\text { provinciales }\end{array}$ & $\begin{array}{l}\text { Organizaciones no } \\
\text { gubernamentales locales }\end{array}$ \\
\hline Ministerio de Desarrollo Humano y Medio Ambiente & Secretaría de Desarrollo Social & Iglesia Bautista \\
\hline Ministerio del Interior & Ministerio de Salud & Iglesia Evangélica \\
\hline ANSES & Ministerio de Educación & Iglesia Católica \\
\hline Instituto Nacional del Aborigen & Ministerio de Obras Públicas & Comisiones vecinales barriales \\
\hline \multirow[t]{5}{*}{ Gerencia Regional de Empleo } & Secretaría de Planificación & Organizaciones comunitarias \\
\hline & Instituto Prov. de Des. Urbano y Vivienda & Consorcios camineros \\
\hline & Subunidad de Prevención para & \multirow[t]{3}{*}{ Centros de jubilados } \\
\hline & Control de la Emergencia & \\
\hline & Administración Provincial del Agua & \\
\hline
\end{tabular}

Fuente: Entrevista realizada a responsables municipales, Marzo 2001. Elab. Propia.

Las modalidades que adquieren las instancias de articulación de actores locales son similares, las que tienen diferentes denominaciones según sea el programa de que se trate. Se identifican como Unidades Ejecutoras Municipales (UEM), Unidades Ejecutoras Locales (UEL), Consejos Sociales Locales (CSL) ó Unidades de Acción Local (UAL). Las que se constituyen en un pre-requisito para el desarrollo local y la descentralización de los programas.

En los municipios en estudio se implementan varios programas nacionales que responden a esta modalidad concertada de gestión, que demandan la creación de Unidades Ejecutoras Locales y la conformación de Consejos Sociales Locales. Esto es replicado por los programas provinciales, cuya modalidad de ejecución se realiza a través de Unidades Ejecutoras Municipales y la creación de las Unidades de Acción Local.

Cuadro 8: Conformación tipo de las UAL, UEM y CSL

\begin{tabular}{lll}
\hline Unidad de Acción Local (UAL) & Unidad Ejecutora Municipal (UEM) & Consejo Social Local (CSL) \\
\hline Intendente & Intendente-Presidente del Concejo & Representante del Municipio \\
\hline Secretario de Gobierno & Concejal Partido Justicialista & Dos representantes por cada grupo solidario \\
\hline Responsable de Acción Social & Representante de Iglesias & Representante de la iglesia local \\
\hline Concejal Partido Justicialista & Jefe Comisaría & Representante del bloque opositor \\
\hline Concejal Partido Acción Chaqueña & Responsable Puesto Sanitario & Representantes ONGs que tengan relación con beneficiarios \\
\hline Concejal Alianza & Representante de Escuelas & \\
\hline Responsable del servicio de salud & \\
\hline Responsable de la región educativa & \\
\hline Representante del Programa AIPO & \\
\hline
\end{tabular}

Fuente: Entrevista realizada a responsables municipales, Marzo 2001. Elab. Propia.

Bajo las diferentes modalidades que según los distintos programas deben adquirir la articulación de actores en el nivel local, se reúnen las mismas personas representando a las mismas instituciones.

Cuadro 9: Las Unidades de Acción Local, Unidades Ejecutoras Municipales y Consejos Sociales Locales en los casos de estudio 


\begin{tabular}{|c|c|c|c|c|c|}
\hline Municipio & $\begin{array}{l}\text { Unidades } \\
\text { Ejecutoras } \\
\text { Locales }\end{array}$ & $\begin{array}{l}\text { Unidades } \\
\text { Ejecutoras } \\
\text { Municipales }\end{array}$ & $\begin{array}{l}\text { Concejos } \\
\text { Sociales } \\
\text { Locales }\end{array}$ & $\begin{array}{l}\text { Unidades de } \\
\text { Acción Local }\end{array}$ & Programas implementados \\
\hline Barranqueras & 2 & 1 & & & UNIDOS PAGV PRONUCHACO \\
\hline Fontana & & 1 & 1 & & UNIDOS Solidaridad PRONUCHACO \\
\hline Puerto Vilelas & & 1 & & & UNIDOS PAGV PRONUCHACO \\
\hline La Leonesa & 1 & 1 & & & UNIDOS PRONUCHACO \\
\hline Pampa del Indio & & 1 & & 1 & UNIDOS AIPO PRONUCHACO \\
\hline Basail & & 1 & & & UNIDOS PAGV PRONUCHACO \\
\hline Colonia Benítez & & 1 & & & UNIDOS PAGV PRONUCHACO \\
\hline Margarita Belén & & 1 & & & UNIDOS PAGV PRONUCHACO \\
\hline
\end{tabular}

Fuente: Entrevista realizada a responsables municipales, Marzo 2001. Elab. propia.

La creación de instancias de articulación y de participación ampliada de actores; de un espacio de democratización de las decisiones; donde se incorporan los intereses y demandas de todos los sectores; y se convierte en un intento de socialización de la información, se ha encontrado con la resistencia de los municipios, quienes visualizan este espacio como injerencia en sus asuntos internos por parte de la oposición, viéndose en algunos casos como una amenaza para las estructuras partidarias que detentan el gobierno local.

Este modelo de gestión se encuentra en la totalidad de los casos observados con las mismas restricciones, los integrantes de las unidades de articulación local cualquiera sea la modalidad que ésta adopte, manifiestan temor a asumir mayores roles que le impliquen nuevas responsabilidades, la escasa capacidad para realizar tareas vinculadas a la autogestión, la poca experiencia para el desarrollo de actividades coordinadas entre las diferentes instituciones, y en este ámbito emergen los conflictos existentes dentro de los miembros de las organizaciones integrantes de la articulación, para lo que no disponen de las herramientas necesarias para una apropiada negociación.

Las asociaciones civiles no son vistas como actores relevantes por las propias áreas sociales para el tratamiento de temas que afectan a las comunidades a la hora de aportar propuestas para introducir cambios orientados a mejorar el nivel de vida de la población. Las organizaciones comunitarias registradas llevan a cabo tareas específicas, colaborando con actividades asistencialitas, las que basan su accionar prioritariamente en el aporte de mano de obra en la implementación de los programas alimentarios, ya que por lo general tienen a su cargo la atención de los comedores infantiles.

Respecto a la existencia de redes municipales, los municipios casos de estudio tienen con relación a la articulación intermunicipal, historias diferentes. Aquellos que pertenecen al Área Metropolitana conforman un espacio de articulación que se hace efectiva y se implementa en situación de emergencia hídrica. Los restantes municipios, están iniciando un proceso de valorización del trabajo conjunto a través de una de las líneas de acción de un Plan de Desarrollo Local, el que persigue como objetivo la creación de redes municipales, formulación de proyectos de gestión asociada de municipios pertenecientes a las mismas micro regiones productivas de la provincia.

\section{Cruce de Variables}

Al realizar el cruce de los valores obtenidos en cada una de las cinco variables analizadas, se obtuvo como producto el nivel de la capacidad de gestión de las áreas sociales municipales en los casos de estudio. Esto nos permitió realizar el siguiente ordenamiento de los casos, según el nivel detectado de su capacidad de gestión, según una lógica de mayor capacidad a menor capacidad de gestión.

En primer lugar y con rango medio-bajo se ubicaron los municipios de Margarita Belén (2.50); segundo La Leonesa (2.48); tercero Fontana y Puerto Vilelas (2.38); cuarto Barranqueras (2.34); quinto Basail (2.30); sexto Pampa del Indio (2.24) y séptimo y último, con un rango bajo, el Municipio de Colonia Benítez (1.74). 
Por lo que podemos constatar que los municipios objeto de estudio respecto de la capacidad de gestión de sus áreas sociales se encuentran en su totalidad en una franja que va desde el rango medio-bajo a bajo. Situándose la mayoría, siete de ocho casos, dentro del rango medio-bajo, y un solo caso en el rango bajo.

Al hacer un promedio de los diferentes casos de estudio acerca de los niveles de capacidad de gestión de las áreas sociales municipales, nos arrojó un valor de 2.29, es decir un rango medio-bajo.

Al realizar el cruce de las variables seleccionadas en el presente trabajo como más relevantes para la determinación del nivel de capacidad de gestión de las áreas sociales municipales, se obtuvo un promedio de los valores alcanzados por cada una de las variables analizadas en los diferentes casos de estudio, con los que se construyó el siguiente orden de prioridad, en el necesario acompañamiento que tanto el estado nacional como provincial deben hacer en este proceso de descentralización de las políticas sociales en manos de los gobiernos locales.

Cuadro 10: Valores promedio de las variables en orden decreciente de prioridad.

\begin{tabular}{|c|c|}
\hline Variables & $\begin{array}{l}\text { Valores } \\
\text { promedios }\end{array}$ \\
\hline La presencia de recursos humanos calificados & 2.10 \\
\hline $\begin{array}{l}\text { El nivel de conformación de las unidades responsables del área } \\
\text { social }\end{array}$ & 2.17 \\
\hline La disponibilidad del recurso financiero requerido & 2.20 \\
\hline \multicolumn{2}{|l|}{ El grado de participación en la planificación, } \\
\hline ejecución y evaluación de programas, proyectos y/o actividades & 2.50 \\
\hline El grado de articulación & 2.50 \\
\hline
\end{tabular}

Fuente: Elaboración propia.

Por lo que finalmente podemos afirmar que, el comportamiento de las variables analizadas nos conduce a concluir acerca de la baja capacidad de gestión de las áreas sociales municipales pertenecientes a los casos de estudio para poder desempeñarse efectivamente en el proceso de descentralización de la política social en que se encuentran inmersas.

\section{REFLEXIONES FINALES}

A partir de los distintos aspectos contemplados en el desarrollo del presente trabajo respecto de las áreas sociales municipales, si bien no es posible establecer un modelo único de gestión, ni con la intención de generalizar las conclusiones arribadas en este reducido universo de casos de estudio, a pesar de las diferencias entre los distintos municipios analizados, es factible vislumbrar necesidades y elementos comunes para el mejoramiento de sus capacidades de gestión pública.

En relación con la presencia de recursos humanos calificados existe una evidente debilidad en las áreas sociales de los gobiernos locales, expresado a través de la situación de carencia que tienen éstas áreas a raíz de la escasa formación de los responsables de dichas áreas y de los integrantes de los equipos técnicos, a su bajo perfil, al reducido número de personas que los integran, a la precariedad de la situación contractual de los mismos y a la falta de experiencia en el desempeño de los roles que le competen.

Si bien en todos los casos de estudio, la totalidad de las áreas sociales tienen presencia institucional en unidades responsables dentro de los gobiernos locales, éstas ocupan un lugar marginal dentro de la estructura organizacional de los Municipios. Si bien las áreas formalmente existen como requerimiento de los programas de financiamiento internacional, no han sido provistas de los 
elementos mínimos necesarios que garanticen un adecuado funcionamiento, es decir no tienen disponibilidad suficiente de equipamiento de tipo informático, de movilidad, de comunicaciones y de un espacio físico apropiado.

Otra característica que limita su capacidad de gestión es la referida a la poca disponibilidad del recurso financiero requerido para el funcionamiento del área social y para la implementación de las acciones. La escasez de los recursos propios ante la creciente demanda de grandes sectores de la población, se suma a la poca gobernabilidad de los recursos existentes de origen externo, el que muchas de las veces no es recibido ni en el tiempo ni en forma prevista.

Se puede afirmar que no existen espacios de planificación de las actividades propias del área, las que en general responden a situaciones de coyuntura, con actividades de tipo asistenciales, con escasas propuestas de acción a excepción de los programas nacionales y provinciales. No existen instancias de monitoreo ni de seguimiento para las acciones; ni tampoco se disponen de bases de información apropiadas para orientar la acción.

Respecto del grado de articulación de las áreas sociales municipales con organismos gubernamentales como no gubernamentales, es incipiente y meramente formal, articulaciones creadas a efectos de cumplimentar los requerimientos exigidos como condición para la implementación de determinados programas sociales de origen externo con financiamiento de la banca internacional. Las áreas sociales municipales no valorizan de manera apropiada la posibilidad de incorporar la participación de la comunidad en el diseño y ejecución de las políticas sociales.

A modo de cierre del trabajo se presentan algunas líneas de acción posibles para una descentralización más eficiente hacia las áreas sociales municipales, las que solamente se mencionan, ya que para su instrumentación requieren de un análisis de mayor profundidad.

Las acciones hacia los gobiernos locales y más precisamente hacia sus áreas sociales, deberían tener prioritariamente tres líneas de acción complementarias entre sí, la capacitación de los equipos técnicos municipales, el fortalecimiento institucional de las unidades responsables de las áreas sociales y la dotación de los recursos financieros necesarios la implementación de la política social a escala local.

\section{BIBLIOGRAFÍA}

TAMAYO SÁENZ, M. 1997. La nueva administración pública, Madrid, Alianza Universidad.

CASTEL, R.

1997. La metamorfosis de la cuestión social. Una crónica del salariado. Buenos Aires, Paidos.

COOK, T.D, REICHARDT, CH. S.

1996. Métodos cualitativos y cuantitativos en investigación evaluativa, Madrid, Ediciones Morata.

CARPIO, J.; NOVACOSKY, I. (COMPS.)

1999. De igual a igual. Brasil, Fondo de Cultura Económica.

REPETTO, F.

1997. Capacidad estatal y políticas frente a la pobreza: los casos de Chile y Argentina (1990-96). México, FLACSO.

MASCAREÑO, C.; BALBI, G.

1995. Descentralización y municipios en América Latina: Necesidades de información de los gobiernos locales. Centro Internacional de Investigaciones para el Desarrollo, Montevideo, CLAD.

AROZENA, J.

1987. Los paradigmas del desarrollo y lo local. Cuadernos del CLAEH N 41 , Montevideo.

SUBIRATS, J.

1998. Análisis de las políticas públicas y eficacia de la administración. Madrid, Instituto Nacional de Administración Pública.

AGUILAR, L. F.

1992. El estudio de las políticas públicas. México, Editorial Miguel Ángel Porrúa.

AGUILAR, L. F.

1992. Problemas públicos y agenda de gobierno. México, Editorial Miguel Ángel Porrúa. 
LLORENS, A. F.

1995. Espacio, territorio y desarrollo económico local. Santiago de Chile.

CORAGGIO, J. L.

1998. Poder local, poder popular. Cuadernos del CLAEH No 45 y 46, Montevideo.

MOREIRA, A.

1998. Hacia un nuevo papel de los Municipios. Cuadernos del CLAEH No 45 y 46, Montevideo. 